\title{
Cloud Migration for SMEs in a Service Oriented Approach
}

\author{
Nicolas Nussbaumer and Xiaodong Liu \\ School of Computing \\ Edinburgh Napier University \\ Edinburgh, United Kingdom \\ x.liu@napier.ac.uk; nnussba@gmail.com
}

\begin{abstract}
Cloud computing has gained immense momentum during recent years and has ultimately become a viable solutions not only for larger firms, but also for small and medium-sized enterprises (SMEs). In order for smaller companies to stay competitive, many have therefore decided in favour of adapting cloud solutions. Given the multitude of issues and challenges that occur during the cloud migration phase, this work proposes a novel framework that helps SMEs to master migration related impediments. In doing this several steps were carried out. Firstly, the work takes into account SME specific requirements and articulates their importance during the cloud provider selection phase. The elicitation was conducted using an extensive literature review, examining case studies, surveys and other publications. The results demonstrate that factors such as security, reliability, cost, performance as well as flexibility and service and support have a pivotal role to play and require close attention. Secondly, decisive attributes were defined that qualify business components and services as cloud-fit. Finally, the framework itself was proposed, which focuses on a systematic service-oriented approach and helps companies to analyse their existing business processes in the course of cloud migration. The framework was verified in its practicability using a concrete scenario and a subsequent prototypical cloud implementation.
\end{abstract}

Keywords - Cloud computing; cloud migration; SOA; SMEs; migration framework.

\section{INTRODUCTION}

Over the last few years cloud computing has experienced a rapid growth in popularity across industries. Enterprises are attracted by cloud offerings, since these are marketed as being superior to on-premise solutions in terms of financial as well as technical dimensions. However, while advantages such as higher cost effectiveness, reliability and scalability are clearly seductive, important factors like the actual migration task are often neglected. Yet, overcoming such migration impediments can become a laborious and costly endeavour, especially for smaller companies with inherently less financial power (Zenga et al., 2010). Despite this fact, current cloud offerings are targeted mainly at the small and medium-sized enterprise (SME) sector, and it is often stated that SMEs in general have a positive attitude towards IT innovation (Liu, 2008) and thus the adoption of cloud computing (Forsman \& Rantanen, 2011; Sultan et al., 2011). It seems inevitable that smaller companies too, will sooner or later have to surmount the challenges associated with the cloud migration. Despite the difficulties, there are currently no major migration approaches or models available that are sufficiently applicable to small and medium-sized companies (Armbrust 2010). Numerous models deal with the economics of cloud adoption while disregarding the equally important migration phase.

This raises the question of what particular steps are required to be taken in order to successfully migrate to the cloud. In order to address this problem, this work aims to devise a comprehensive framework for cloud migration, especially tailored to meet SME specific requirements. The framework focuses on the analysis and eventual migration of business processes, by means of the service-oriented paradigm In this model economic factors are considered to be out of scope.

In the context of this work it will first be necessary to examine the different facets of cloud computing as well as assessing the unique characteristics of small businesses. Special attention will also be devoted to the concept of Service Oriented Architecture (SOA), since the framework to be developed significantly relies on this paradigm.

On the basis of this extensive background research, this study will then elicit requirements pertaining specifically to SMEs that need to be taken into account during cloud migration exercises. In fact, the requirements to be gathered not only aim to help in decisions about which services to migrate, but will also help in finding the most suitable cloud provider. Based on these requirements, the migration framework will then be developed.

Finally, the framework's practicability and effective applicability will be verified based on a sample business process, which will go through every stage of the framework. This process will eventually be implemented and deployed using a cloud-based platform.

The rest of this paper is organized as follows. Section II critically analyses the related work. Section III discusses the requirements of SME cloud computing we collected. In Section IV, an SOA based cloud migration framework is proposed for SMEs. Section V illustrates the framework and 
its implementation with a real-life case study. Finally, Section VI presents the conclusions and the future work.

\section{RELATED WORK}

The foremost drivers for cloud computing are consequential cost advantages. Due to the limited financial resources of SMEs, compared to their larger counterparts, the cost factor seems to be even more crucial for these smaller companies (Chien \& Chien, 2010; Sultan, 2011). Given their limited financial power, such companies cannot undertake large investments and therefore face significant advantages in adopting the cloud, as there is little or no upfront investment necessary (Daneshgar et al., 2011). The enterprise therefore gets to turn their capital expense into a variable operating expense (Khanapurkar, 2011). This fact is especially important for SMEs that have neither the required capital nor the willingness to afford expensive IT infrastructure. Additionally, whereas large corporations need to operate and manage expensive legacy systems, SMEs are less likely to have such major infrastructure, hence the overall costs can be potentially lower (Grabova et al., 2010). On the other hand, it could be argued that the absence of such complex enterprise applications and IT infrastructure even facilitates cloud service integration (Khanapurkar, 2011).

Cloud computing is seeing an increasing attention that will inevitably drive businesses to migrate existing on-premise applications onto the cloud (Peddigari, 2011). Even though this attractive proposition has widespread acceptance among SMEs, the numbers of companies actually utilising the cloud appear to be rather low (Truong, 2010). There are a variety of reasons that can account for to this discrepancy between the optimistic predictions in literature and actual cloud implementations. In general, security concerns are among the top obstacles for adopting the cloud (Matuszak \& Lamoureux, 2011). However, beside security studies have found that the actual migration of existing applications and processes poses major challenges for SMEs (Cloud30, 2012; Catteddou \& Hogben, 2009). Khajeh-Hosseini et al. (2012) confirms this, warning that the adoption of cloud computing in enterprise environments is not a trivial undertaking, while Peddigardi (2011) points out that the challenges are often due to a lack of understanding of the business processes itself. Many SMEs therefore fail to fully comprehend the intricacies of their enterprise application landscape (Golden, 2009; Hajjat et.al, 2010). As a result, academics are starting to show interest in the migration problem, aiming to help organisations cope with the difficulties of embarking on the cloud. The majority of authors, however, mainly focus on the financial perspective of cloud adoption as the following section indicates.

Klems et al. (2009), for instance, present a framework that can be used to compare the cost of using the cloud with more conventional approaches, such as in-house IT infrastructure. This cost-based decision framework enables decisions to be made whether or not to deploy applications to the cloud. However, one drawback of this examination centres on the fact that their framework was at an early development stage, no concrete results were provided. Furthermore, the framework emphasises economic factors of the cloud, and does not consider the concrete migration steps that have to be taken.

Khajeh-Hosseini et al. (2010b) conducted a cost analysis based on a case study and found that there were significant cost advantages when migrating to cloud services such as Amazon EC2. The research did not produce a framework, but rather it analysed infrastructure costs and highlighted the risks and benefits of cloud computing. Despite its already narrow economic focus, the study also disregarded the cost of doing the actual migration work. In a subsequent study, Khajeh-Hosseini et al. (2012) therefore present the "Cloud Adoption Toolkit" that facilitates comprehensive infrastructure cost modelling while assessing socio-technical impacts, such as organisational change, by means of a stakeholder analysis. Despite the broad application of tools, including the "Technology Suitability Analysis", "Cost Modelling \& Energy Consumption Analysis" and "Stakeholder Impact Analysis", this framework also has its drawbacks: it aims specifically at addressing the challenges of cost analysis and can therefore be seen only as a strategic, high-level tool that represents a "promising starting point for cloud migration decision making"; additionally, in spite of incorporating a technology suitability analysis, the framework does not stipulate any particular methodology as to how to transfer existing services onto the cloud.

A more systematic approach towards cloud migration was achieved with the "Goal- oriented Requirements Engineering Approach" proposed by Zardari \& Bahsoon (2011), which views cloud procurement and adoption from a requirements engineering perspective. By doing so, the proposed solution represents a systematic methodology that helps stakeholders in negotiating requirements against cloud services provision. In terms of cloud adoption this can certainly be considered an important step towards migrating assets to the cloud. The disadvantages of this approach can be identified in its limited scope. As the name suggests, it focuses on requirements aspects only and can therefore be seen as a part of an overarching cloud migration approach. Hence, it does not suggest any methodology for analysing existing business processes, as a step towards successful cloud migration.

Hajjat et al. (2010) suggest another noteworthy approach towards embarking upon the cloud. The study focuses on the beneficial cloud migration within hybrid cloud architectures. By doing so, enterprise application components are analysed based on transaction and security parameters, allowing a decision on whether or not the component is suitable for the cloud. Although it has contributed a lot to an understanding of the issues of cloud migration, this model has some drawbacks. Firstly, the study does not culminate in a workable, well-structured cloud migration practice that can be universally applied as a framework. Secondly, the approach is geared towards large enterprise applications that require in-depth component analysis, complex transaction delay calculations and security configurations making it unsuitable for smaller organisations.

In summary, cloud computing no longer remains a far-fetched paradigm: rather it is a tangible solution that SMEs have to cope with in order to stay competitive (Buyya 2009). 
Despite the necessity for SMEs to embark on cloud computing, the associated migration poses major obstacles that are often difficult if not impossible to overcome if not applying an appropriate, systematic approach. Nevertheless, this study of the literature has shown that a workable framework for migrating existing SME specific business services to the cloud has not yet been discussed or developed to any significant extent. So far, migration approaches have been left to industry practitioners therefore becoming closed proprietary tools that are often accompanied by expensive consultancy costs. As seen above, the majority of non-proprietary frameworks either have their focal point on cost aspects (so-called "cloudonomics") that allow strategic decision-making based on Cost Benefit Analysis (CBA), or lack sufficient SME relevance. The only model identified that bears some relevance in terms of applying service-oriented aspects, is the one proposed by Linthicum (2010).

Given the absence of any practicable SME specific cloud migration methodology, the proposed solution aims at creating an exemplary framework addressing the needs of smaller businesses. This framework applies a service-oriented approach whilst taking SME centric requirements into consideration. The benefit of this methodology lies in the fact that it concentrates on the unique capabilities of SMEs, ensuring that their existing processes are understood so sustainable cloud migration decisions can be made. Moreover, a service-oriented approach supports agility - the ability to change business processes quickly - in order to react swiftly to an ever-changing business environment.

\section{SME ClOUd COMPUTING REQUIREMENTS}

Six SME cloud objectives that have a significant influence on SMEs' requirements for cloud provider selection were extracted. Each factor represents an objective that SMEs seek to fulfil when undertaking cloud adoption, while at the same time driving companies' cloud strategy. The "Six Cloud Objectives" are presented in Figure 1.

\begin{tabular}{|c|c|c|c|c|}
\hline Cost & Flexibility & Performance & Security & Reliability \\
Requirements \\
Support
\end{tabular}

The literature sources we reviewed were analysed to obtain the requirements for SME business services to be cloud-fit. The IT services supporting business processes that are most likely to be cloud-sourced, according to this analysis, are listed in Table 1. The results indicate that services such as "CRM \& Sales Management", "Application Development" and "Project Management" are most likely to be migrated to a cloud provider. Conversely services such as "HR Management", "ERP”, "E-Procurement" and "Accounting \& Finance" are predominantly operated in-house.

\begin{tabular}{|c|c|}
\hline Likelihood & SME Cloud Business Services/ Applications \\
\hline H & Application Development \\
\hline H & CRM \& Sales Management \\
H & Email \& Communication \\
\hline
\end{tabular}

\begin{tabular}{|c|c|}
\hline H & $\begin{array}{c}\text { Office Applications } \\
\text { M }\end{array}$ \\
M & Collaboration Tools \\
M & Payroll \\
L & Project Management Software \\
L & Accounting \& Finance \\
L & E-Procurement \\
L & ERP \\
& Human Resource Management (HR) \\
& Th]igh; [M]edium; [L]ow
\end{tabular}

The results given above imply some common, overarching characteristics among these cloud-sourced business processes from which the following features can be derived. These are:

- Standardised. The business applications are well defined and widely accepted across industries (i.e. best-practice), therefore do not require extensive customisation (Leavitt, 2009). Moreover, such a wide-established customer base allows companies to consult reference implementations during decision-making.

- Streamlined. The previously mentioned business applications are highly optimised, aiming for efficiency and effectiveness, thus acting as business catalysts while reducing unnecessary overheads.

- Non-business critical. The business applications are not mission-critical, i.e. core processes, in their nature, rather, they can be regarded as commodities that allow SMEs to focus on their core competencies as well as innovation processes.

- Non-sensitive data. The business applications do not entail sensitive data, such as trade secrets, classified or proprietary information, and therefore do not aim to safeguard customer data (e.g. Email \& Communication, Office Applications and CRM \& Sales Management).

Given these four basic characteristics of SME business applications, meaningful insights into the rationale of SMEs' cloud adoption can be gained. At first sight, these characteristics might come as surprise, given the purportedly high willingness of SMEs to swiftly adopt new cloud services. It seems that, contrary to popular belief, SMEs tend to rely on well-established cloud services such as CRM, communication, office applications or application development services rather than on highly business critical applications. Arguably, SMEs can be seen as pragmatists rather than early adopters of cloud offerings.

Nevertheless, the features elicited so far do not exhibit a sufficiently profound level of detail that would help to answer the question of which component to migrate to the cloud. In the course of this research it is therefore necessary to analyse the underlying services that constitute business applications. In this respect, the SOA paradigm can help to define service prerequisites that need to be fulfilled in order for a service to become cloud-fit.

- Loosely Coupled. For each service to become cloud-fit, it should leverage a loosely coupled architecture, in which dependencies between services are to a great extent 
reduced or even eliminated. Such less tied services reduce the risk that a change in one service (i.e. module or component) will force a change in another service (Linthicum, 2010). The notion of loose coupling necessitates high modularity of each service, in such way that it exists, in the form of self-contained modules that can be bound together to form other, perhaps more advanced services (Sneed, 2006).

- Well-Defined Integration Points. In order for this coupling to work, well-defined interfaces are imperative for every potential cloud-fit service. Processes that are placed on the cloud need to have appropriately defined interfaces (i.e. APIs) that allow ease of integration with existing on-premise systems. Not only does this enable seamless collaboration between in-house and cloud services but also creates a holistic architecture that facilitates information flow without obstacles (Rimal, 2011).

- Reusable. Another important requirement is the service reusability. When a service encapsulates business logic that is useful not only for one consumer (requester), it is considered reusable. Reusability is a fundamental concept of SOA that also applies to cloud computing, in that it allows multiple requesters to use the service at the same time in a different context (IBM, 2012).

- Self-Descriptive. Reusability requires self-descriptive service interfaces that consistently adhere to their service description or formal contract. Also referred to as "Modular Understandability", it allows consumers to understand the function of the service without needing to understand other services (McGovern, 2003).

- Thin Client Interface. Another requirement for such candidate services is the fact that they are being operated over the Internet, hence using the Web Browser as the primary vehicle for interaction. To date, most services offered, in particular SaaS models, exclusively use the Browser as User Interface (UI) in order to facilitate platform independency (Miller, 2008). Thus, Rich Internet Applications (RIA) have a pivotal role to play in todays cloud applications, since they allow dynamic behaviour and increased user experience (Linthicum, 2010).

- Novelty of Service. It is commonly agreed upon that new applications, including processes, services and data, are generally simpler to migrate to the cloud than legacy systems. This is because legacy systems typically need to be partially or even completely rewritten from scratch in order to successfully live on the cloud and work as distributed services (Linthicum, 2010).

\section{Cloud Migration FrameWORK}

A framework for migrating existing on-premise business processes using a service oriented approach is developed. The framework advocated here provides a generic methodology to analyse existing SME business processes and migrate them to the cloud by considering important, SME specific cloud requirements. An overall view of the framework is shown in Figure 2. It entails the following components:
- Business Process Decomposition: The main part of the cloud migration framework is subsumed under the term business process decomposition, which entails processes, services, and data to be analysed.

- Process: The process is the starting point of the framework and describes SME business processes that are being further analysed.

- Service: The service constitutes a business process and offers small-grained, self-contained services to be used by a consumer. In this step the different services that build a business process are being analysed.

- Data: Every service creates, reads, updates, deletes (CRUD), and therefore relies upon data. In this step the underlying data of a particular service is being analysed.

- Service Candidates: This pool of candidates consists of services that fulfil the "SME Service Requirements" for cloud migration and therefore are regarded as cloud-fit.

- Platform Selection: This selection process aims to choose a suitable cloud provider as well as service model that fulfils the "SME Cloud Requirements".

- Deployment: Finally, the framework culminates in the deployment of the selected services to the cloud.

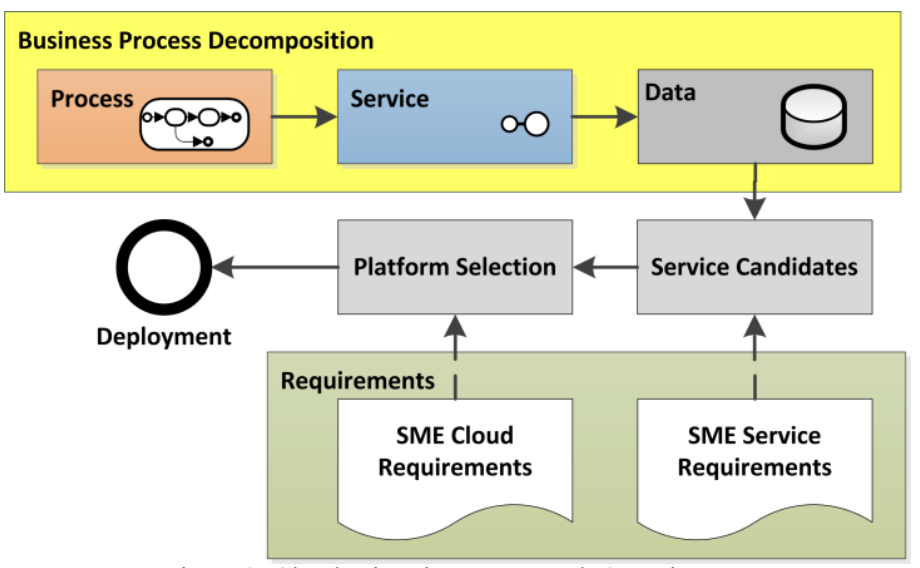

Figure 2. Cloud Migration Framework Overview

\section{A. Business process decomposition}

This part of the framework is responsible for the service-oriented decomposition of business processes that are potentially migrated to the cloud. The process will be broken down into a subset of services, which will further be dismantled into the underlying data, allowing a fine-grained analysis of the existing SME business process. Using the SOA paradigm and its associated notion of application composition, the methodology has been developed as illustrated in Figure 3.

\begin{tabular}{|c|l|}
\hline \multicolumn{1}{|c|}{ Abstraction Level } & \multicolumn{1}{|c|}{ Step } \\
\hline Process & $\begin{array}{l}\text { 1. Identify Problem Domain } \\
\text { 2. Create Process Model }\end{array}$ \\
\hline Service & $\begin{array}{l}\text { 3. Identify Unerlying Services } \\
\text { 4. Create Service Repository }\end{array}$ \\
\hline Data & $\begin{array}{l}\text { 5. Identify Data } \\
\text { 6. Create Data Dictionary \& Model }\end{array}$ \\
\hline
\end{tabular}

Figure 3. Business Process Decomposition 


\section{B. $\quad$ Service candidates}

The pool of service candidates comprises of the decomposed processes, services and the associated data. At this stage of the framework, the services identified in the prior steps are collected and compared against the requirements gathered in section 3. For reasons of clarity, these distinct characteristics are summarised in Table 2.

\begin{tabular}{|l|l|}
\hline & Requirement \\
\hline \multirow{3}{*}{$\begin{array}{l}\text { Business Application/ } \\
\text { Process }\end{array}$} & Streamlined \\
& Non- business critical \\
& Non- sensitive data \\
& Loosely Coupled \\
\hline Well-defined Integration Points \\
\hline Service & Reusable \\
\hline & Self-descriptive \\
& Thin client interface \\
& Novelty of service \\
\hline \multicolumn{1}{|c|}{ Table 2. Business Application/ Process and Service Requirements }
\end{tabular}

Services, which exhibit attributes that match the features shown in Table are geared for cloud migration and can therefore be declared as cloud-fit.

\section{Platform selection}

This step of the migration framework selects the appropriate platform that best suits a company's business requirements. By doing so, it provides assistance in selecting adequate:

- cloud service models,

- cloud delivery models and

- cloud providers that suit the specific needs of an SME.

The requirements gathered in section 3 build a solid foundation for the cloud selection exercise. Table 3 summarises these classes of requirements that need to be taken into account in provider selection.

\begin{tabular}{|c|c|c|}
\hline $\begin{array}{l}\text { Cloud } \\
\text { Objective }\end{array}$ & $\begin{array}{l}\text { Abstract Categorisation } \\
\text { Criteria }\end{array}$ & Requirement \\
\hline \multirow{6}{*}{ Cost } & \multirow{5}{*}{ Pricing } & Price Model \\
\hline & & Price Transparency \\
\hline & & Price Stability \\
\hline & & Payment Plans \\
\hline & & Payment Methods \\
\hline & Service Billing & Billing Granularity \\
\hline \multirow{7}{*}{ Flexibility } & \multirow{4}{*}{ Portability } & Standardisation \\
\hline & & Interfaces (API) \\
\hline & & Data Portability \\
\hline & & Interoperability \\
\hline & \multirow{3}{*}{ Resources } & Scalability \\
\hline & & Customisation \\
\hline & & Installation Time \\
\hline Performance & Infrastructure & Transaction Speed \\
\hline
\end{tabular}

\begin{tabular}{|c|c|c|}
\hline & & Storage Capacity \\
\hline & & Transfer Volume \\
\hline & & Computing Power \\
\hline & Tochnoloon & Load Balancing \\
\hline & 1ecnnorogy & Multi-Tenancy \\
\hline Security & Data Centre & Hardware \\
\hline & & Software \\
\hline & Network & Connection \\
\hline & Data Protection & Data Protection \\
\hline & & Compliance \\
\hline Reliability & Reliability & Disaster Recovery \\
\hline & & Redundancy \\
\hline & Service Level Agreement & Availability \\
\hline & & Liability \\
\hline & Trust & $\begin{array}{l}\text { Service } \\
\text { Transparency }\end{array}$ \\
\hline & & Reporting \\
\hline Service \& & User Interface & Customisation \\
\hline Support & & Usability \\
\hline & Service Management \& & Support \\
\hline & Operation & Controlling \\
\hline & & Monitoring \\
\hline & & $\begin{array}{l}\text { System } \\
\text { Management }\end{array}$ \\
\hline
\end{tabular}

\section{V.CASE STUDY}

The case study aims to verify the proposed cloud migration framework. A scenario business process was thereby selected and decomposed into services and data, based on the cloud migration framework. In addition to the decomposition of the process, a cloud implementation was pursued which culminates in a practical and workable cloud-based prototype.

\section{A. Application of the migration framework}

\section{1) Process}

\section{Identify Problem Domain}

Sales Management applications are popular among SMEs and in this context highly suitable for demonstration purposes. A rudimentary, yet meaningful part of such sales management applications is the process of creating a customer sales order, henceforth referred to as "Create Customer Sales Order". This particular business process has therefore been chosen for further analysis.

\section{Create Process Model}

Figure 4 illustrates a simplified "Create Customer Sales Order" process using the BPMN 2.0 standard. The process comprises the four tasks "Check Customer", "Create Customer Record", "Check Product Availability" and "Record Sale" as well as the sub process "Order Product". 

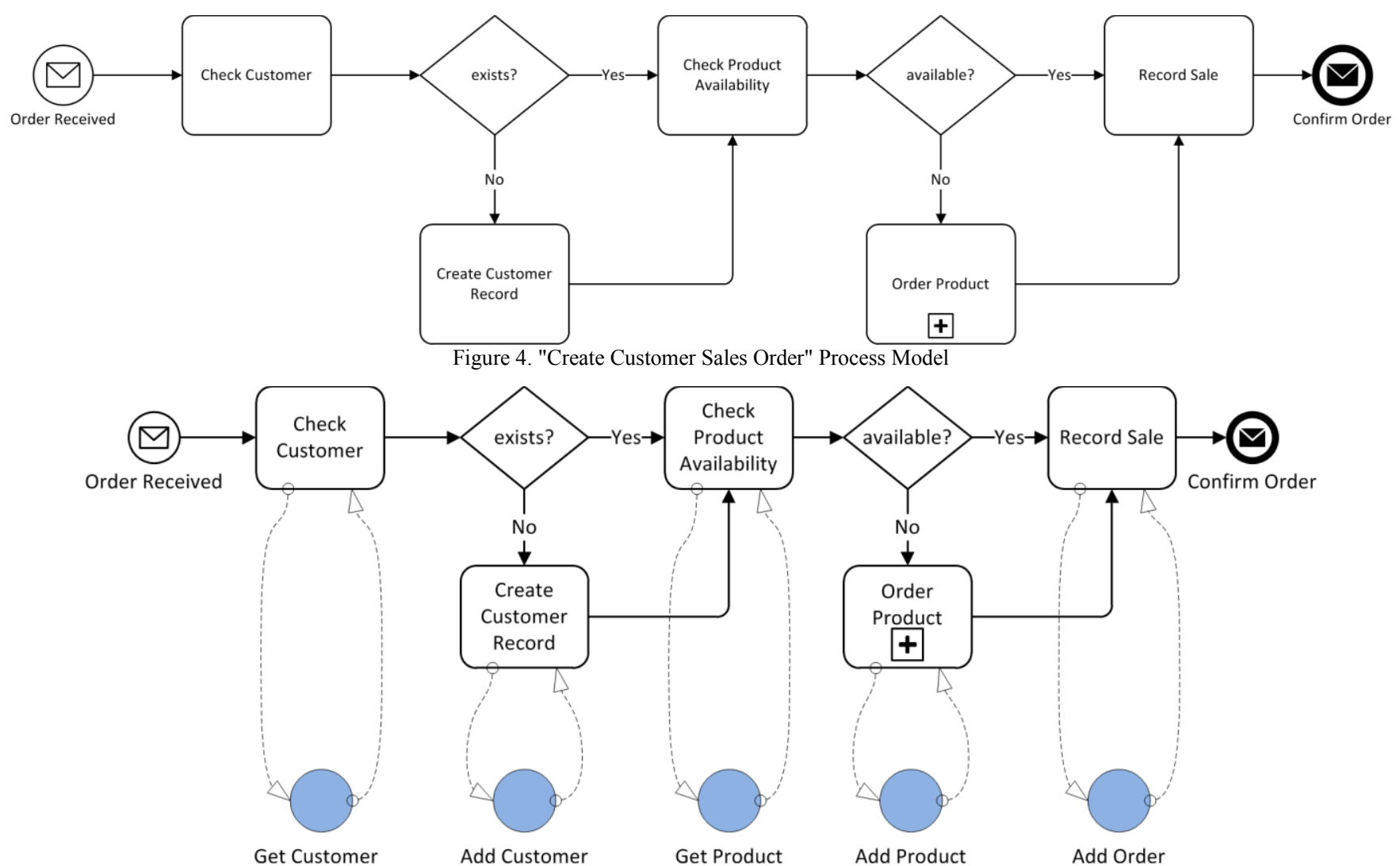

\section{2) Service}

\section{Identify Underlying Services}

Figure 5 depicts process of mapping the process and its tasks to the corresponding underlying services. In this simplified example, each task contains one single subjacent service that fulfils self-contained functionality.

In Table 4 the decomposition of the business process "Create Customer Sales Order" into each service is further illustrated.

\begin{tabular}{|c|c|c|}
\hline Process & Task & Service \\
\hline \multirow{5}{*}{$\begin{array}{l}\text { Create Customer Sales } \\
\text { Order }\end{array}$} & Check Customer & Get Customer \\
\hline & $\begin{array}{c}\text { Create Customer } \\
\text { Record }\end{array}$ & Add Customer \\
\hline & $\begin{array}{l}\text { Check Product } \\
\text { Availability }\end{array}$ & Get Product \\
\hline & Order Product & Add Product \\
\hline & Record Sale & Add Order \\
\hline
\end{tabular}

\section{Create Service Repository}

Table 5 represents the service repository that captures and describes the 1 out of the 5 services elicited in the prior step as an example. The "Performance Attributes" property is intentionally left blank, since for the purpose of this example, performance parameters can be regarded as irrelevant.

\begin{abstract}
Name
Add Customer
\end{abstract}

Description

Revision

Adds a customer record (object) to the system.

Interface

Definition 1.0

Authentication

Performance

Attributes

Security

Parameters

Encryption $=$ none , Credentials $=$ Username,

Dependencies

Password

$$
\text { Table 5. "Create Customer Sales Order" Service Repository }
$$

\section{Data, service candidates and platform}

Data related to the services was first identified, and then it is necessary to record and document them in the data dictionary. Most appropriate services were selected according to the criteria defined in section $4 \mathrm{~b}$. A solution that satisfies the requirements and suits the demonstration purpose is VMware's Cloud Foundry, an open PaaS that supports various rapid application development (RAD) frameworks.

\section{B. Implementation}

Having decomposed the "Create Customer Sales Order" process into services and data, the exemplary business process was then implemented. The Spring Roo software tool has been 
utilised providing a RAD environment based on Java enterprise software. Furthermore, in accordance with the requirements to realize a cloud-enabled solution, the VMware MCF platform has been chosen for deployment of the application.

This view of Kruchten's 4+1 Architectural Model describes the actual deployment of the system. Figure 6 shows the deployment concept of this prototypical implementation.

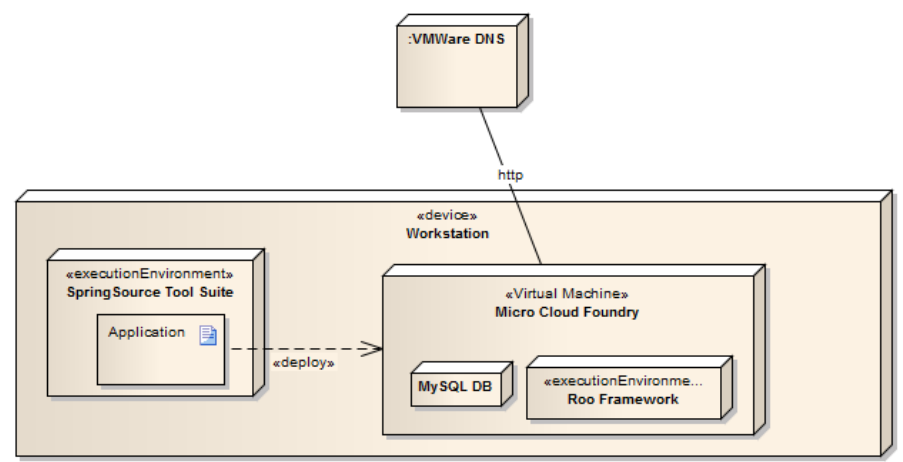

Figure 6. Application Deployment Model

In the light of this software prototype the "SpringSource Tool Suite" (STS) has been utilised as development environment (i.e. "executionEnvironment"). STS facilitates simplified application deployment by integrating MCF as a native server instance. MCF on the other hand runs as a "Virtual Machine" instance on the same "Workstation" as STS, incorporating MySQL database services for data persistence. Finally, the external node "VMWare DNS" is responsible for resolving the unique URL, which is required for accessing the deployed application through the Browser.

\section{ROO}

\begin{tabular}{|c|c|}
\hline COMPONENT & Welcome to SMESales \\
\hline Create new Component & \multirow{5}{*}{$\begin{array}{l}\text { Welcome to SMESales } \\
\text { Spring Roo provides interactive, lightweiç } \\
\text { delivery of high performance enterprise } J\end{array}$} \\
\hline List all Components & \\
\hline FRAME & \\
\hline Create new Frame & \\
\hline List all Frames & \\
\hline CUSTOMER & \\
\hline Create new Customer & \multirow[t]{8}{*}{ Home | Language: 昜囷 | Theme: standard I } \\
\hline List all Customers & \\
\hline BICYCLE & \\
\hline Create new Bicycle & \\
\hline List all Bicycles & \\
\hline SALES ORDER & \\
\hline Create new Sales Order & \\
\hline List all Sales Orders & \\
\hline
\end{tabular}

Figure 7. Prototype "Home" Screen

\section{CONCLUSIONS AND FUTURE WORK}

Although cloud computing has gained momentum during the last five years, many small and medium-sized enterprises are still at the beginning of a cloud transition phase during which they face a myriad of challenges. However, there are currently no mature techniques or frameworks available to address issues of migrating business processes to the cloud using a SME centric focus. This study aimed at devising a systematic migration methodology specifically geared towards small and medium-sized enterprises. The study found that companies need to adequately understand their existing business processes in the course of a successful cloud migration. Hence, a systematic service-oriented approach was introduced that helped analyse business processes, its underlying services and data. Furthermore, the study proposed business service specific requirements in order to decide whether or not a certain component is qualified for cloud migration. And finally, during the platform selection phase, additional SME distinct requirements were proposed that assisted in finding an appropriate cloud solution.

The main contribution of this work is the Cloud Migration Framework, which can be regarded as a step towards overcoming cloud migration impediments. Its novel combination of SME distinct needs, business service attributes and the service-oriented approach ensures that the focus remains on the unique strengths of the respective company. This is a crucial aspect for SMEs to stay competitive and successfully cope with an increasingly changing business environment.

At present, the migration methodology does not consider the economic factors of the cloud migration. For the framework to become a fully-fledged tool that covers the whole migration life cycle, it is necessary to complement the model with economics-driven approaches that assess and evaluate the cost effectiveness of the migration. Furthermore, for future research, the framework will include concrete metrics for measuring cloud-fit services against the prerequisites such as loose coupling, reusability or well-defined interfaces.

\section{REFERENCES}

[1] Armbrust, M., Fox, A., Griffitz, R., Josepf, A.D., Katz, R., Konwinski, A., Lee, G., Patterson, D., Rabkin, A, Stoica I., Zaharia. M. (2010). A View of Cloud Computing. Communications oft he ACM, 53(4), 50-58.

[2] Buyya, R., Yeo, C. S., Venugopal, S., Broberg, J., \& Brandic, I. (2009). Cloud computing and emerging IT platforms: Vision, hype, and reality for delivering computing as the 5th utility. Future Generation Computer Systems, 25(6).

[3] Catteddu D., Hogben, G. (2009). An SME perspective on Cloud Computing. Survey. Retrieved March 10, 2012, from: http://www.enisa.europa.eu/.

[4] Chien, C. S., \& Chien, J. (2011). Insight to Cloud Computing and Growing Impacts, Information Computing and Applications, Springer, Vol 105, pp 250-257.

[5] Cloud30. (2012). The Cloud: Why so many SMEs are Reluctant. Retrieved March 29, 2012 from: http://www.cloud30-computing.com.

[6] Daneshgar, F., Worasinchai, L., \& Low, G. (2011). An Investigation of 'Build vs. Buy'. Decision for Software Acquisition in Small to Medium Enterprises. Conference on Interdisciplinary Business Research.

[7] Forsman, H., and Rantanen, H. (2011). Small manufacturing and service enterprises as innovators: a comparison by size. European Journal of Innovation Management, 14(1), 27-50.

[8] Grabova, O., Darmont, J., Chauchat, J.-H., \& Zolotaryova, I. (2010). Business intelligence for small and middle-sized enterprises. SIGMOD Record, 39(2), 39-50. 
[9] Hajjat, M., Sun, X., Sung, E.Y., Maltz, D., Rao, S.,Stripanidkulchai, K and Tawarmalani, M. (2010). Cloudward Bound: Planning for Beneficial Migration of Enterprise Applications to the Cloud. ACM SIGCOMM, 243-254.

[10] IBM (2012). How SOA can ease your move to cloud computing. Retrieved April 15, 2012 from: http://www-01.ibm.com/software/solutions/soa/newsletter/nov09/article _soaandcloud.html.

[11] Khajeh-Hosseini, A., Greenwood, D., \& Sommerville, I. (2010b). Cloud Migration: A Case Study of Migrating an Enterprise IT System to IaaS. 3rd International Conference on Cloud Computing (pp. 450-457). Washington: IEEE Computer Society.

[12] Khajeh-Hosseini, A., Greenwood, D., Smith, J. W. and Sommerville, I (2012), The Cloud Adoption Toolkit: supporting cloud adoption decisions in the enterprise. Softw: Pract. Exper. , 42(4), 447-465.

[13] Khanapurkar, N. (2011). The Cloud: Changing the Business Ecosystem. Retrieved March 16, 2010 from: http://www.kpmg.com/IN/en/IssuesAndInsights/ThoughtLeadership/The _Cloud_Changing_the_Business_Ecosystem.pdf.

[14] Klems, M., Nimis, J., and Tai, S. (2009). Do Clouds Compute? A Framework for Estimating the Value of Cloud Computing. Markets, Services, and Networks, Lecture Notes in Business Information Processing, vol. 22.

[15] Leavitt, N. (2009). Is Cloud Computing Really Ready for Prime Time? Computer 42(1). 15-20.

[16] Linthicum, D. S. (2010). Cloud computing and SOA convergence in your enterprise: A step-by-step guide. Upper Saddle River, NJ: Addison-Wesley.

[17] Liu, X. and Ye, H. (2008). A Sustainable Service-Oriented B2C Framework for Small Businesses. 4th IEEE International Symposium on Service-Oriented Systems Engineering (SOSE'08), Taiwan, Dec 2008.
[18] Matuszak, G. and Lamoureux, T. (2011). Embracing the Cloud: Global forces shaping the service provider market. [Electronic Version] Retrieved March 14, 2012 from: http://www.kpmg.com/Global/en/IssuesAndInsights/ArticlesPublication s/Documents/embracing-cloud.pdf.

[19] McGovern, J. (2003). Java Web services architecture. San Francisco: Morgan Kaufmann Publishers.

[20] Miller, M. (2008). Cloud computing: Web-based applications that change the way you work and collaborate online. Indianapolis, Ind: Que.

[21] Peddigari, B.P. (2011). Unified Cloud Migration Framework - Using factory based approach. India Conference (INDICON), 1-5.

[22] Rimal, B. P., Jukan, A., Katsaros, D., \& Goeleven, Y. (2011). Architectural Requirements for Cloud Computing Systems: An Enterprise Cloud Approach. Journal of Grid Computing, 9(1), 3-26.

[23] Sneed, H. M. (2006). Integrating Legacy Software into Service Oriented Architecture. Proceedings of the Conference on Software Maintenance and Reengineering.

[24] Sultan, N. A. (2011). Reaching for the "cloud": How SMEs can manage. International Journal of Information Management, 31(3), 272-278.

[25] Truong, D. (2010). How Cloud Computing Enhances Competitive Advantages: A Research Model for Small Businesses. The Business Review, 15(1), 59-65.

[26] Zardari, S., Bahsoon, S. (2011). Gloud adoption: A Goal-oriented Requirements Engineering Approach. SECLOUD '11. Proceedings of the 2nd International Workshop on Software Engineering for Cloud Computing, 29-35.

[27] Zenga, S. X., Xie, X. M., \& Tam, C. M. (2010). Relationship between cooperation networks and innovation performance of SMEs. Technovation, 30(3), 181-194. 